Research Article

Andrei Ignatov* and Andrei Gorchakov

\title{
Tool for Simulating Branch and Bound Computations
}

https://doi.org/10.1515/comp-2020-0115

Received Dec 03, 2019; accepted Feb 16, 2020

\begin{abstract}
The paper describes a simulator of parallel Branch and Bound (BnB) method. Several subdomain trees for benchmark functions are analyzed, a characteristic Gaussian-like distribution is discovered. An algorithm of artificial tree generation is formulated according to this criterion. The process of simulator modeling is described, several computational experiments are conducted. Their results show a hyperbolic decrease trend for modeled time as the number of computational units grows, which is concluded to be similar to real systems.
\end{abstract}

Keywords: Branch and Bound, Simulator, Optimization

\section{Introduction}

Branch and Bound (BnB) method [1] is a widely used technique for solving optimization problems. It performs recursive split of the examined area evaluating consistence of further processing of each part. As a result, a subproblem tree is built. Parts of the tree can be handled independently, which makes the algorithm suitable for parallel processing. Multiple investigations on applications and scalability of this algorithm have been made. A system for solving global optimization problems with Branch and Bound method was proposed in [2]. Research on processing nonlinear inequalities was made in [3]. Some research on complexity and scalability of this method was made in $[4,5]$.
A system utilizing this method may include great amounts of computational resources that include not only single computers but also clusters of multiple machines [6] and 'virtual' supercomputers consisting of volunteer computing resources [7]. However, testing of such system may require long periods of time, which is the main cause for simulating Branch and Bound method. Simulation of this algorithm is complicated with the fact that the subproblem tree is not known in advance. Analysis of the existing tools revealed the absence of parallel Branch and Bound simulators, though there are tools based on discrete events that work well for most situations. One of these systems is ROSS (Rensselaer's Optimistic Simulation System) [8]. It is a parallel simulator launched on multiprocessor systems or supercomputers which has a mapping between modeled processes and the ones of the real computational system.

Another toolset called SimGrid [9] provides functionality for developing simulators for distributed systems and applications. It is worth noting that SimGrid is limited on this architecture, so modeling of realtime multiprocessor systems appears to be impossible [10]. At the same time, SimGrid has an advantage of following a timeline for every computational node, which makes it possible to evaluate time shared by all processes and consider tasks that are closest to finish. Apart from that, this tool also supports using discrete events.

Despite the advantages, the described tools do not take into account the specific features of $\mathrm{BnB}$ method, and therefore are hardly applied to modeling parallel BnB. Thus, it was decided to develop a new tool for modeling Branch and Bound computations.

\section{Building Subproblem Trees}

\footnotetext{
^Corresponding Author: Andrei Ignatov: National Research University Higher School of Economics, Moscow, Russia; Federal Research Center "Computer Science and Control” of Russian Academy of Sciences, Moscow, Russia; Email: rayignatov@outlook.com Andrei Gorchakov: Federal Research Center "Computer Science and Control" of Russian Academy of Sciences, Moscow, Russia; Moscow Institute of Physics and Technology, Dolgoprudnyi, Moscow oblast, Russia; National Research University Higher School of Economics, Moscow, Russia; Email: andrgor12@gmail.com
}

To generate trees similar to the real ones, we analyzed structure of subproblem trees for several functions from the unconstrained optimization benchmark set [11]. For every problem, numbers of tasks on different depths were collected. Corresponding diagrams for three functions are presented in Figure 1. As seen from graphs, all curves can be interpolated with Gauss functions, which leads to an idea

¿ Open Access. (C) 2020 A. Ignatov and A. Gorchakov, published by De Gruyter. 

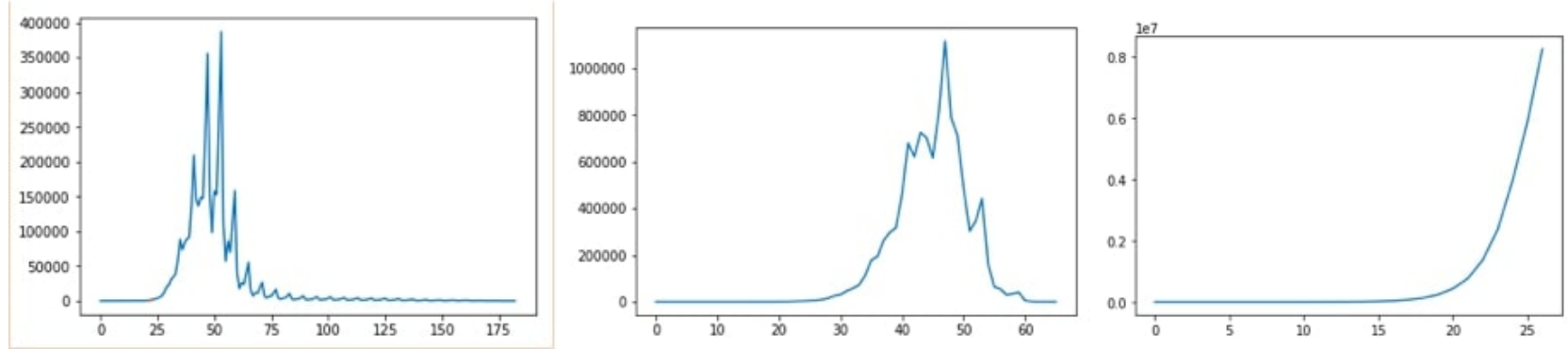

Figure 1: Numbers of tasks in different depths of trees for problems: a) BiggsEXP5; b) BiggsEXP6; c) Cluster2D2

about typical distribution of subproblem tree widths. This result turned out to be the same for all analyzed functions.

Therefore, in tree generation algorithm, widths of created trees should be distributed normally. For tree generation, we used an approach of recursive tree building with evaluation of probability for a further split. The condition for creating child tasks of the current one is set as follows

$$
\text { random }(0,1)>F_{\text {prob }}(\text { cur_depth/max_depth }),
$$

where cur_depth is the current tree depth, max_depth is the maximal depth set in initialization, and $F_{\text {prob }}$ is a certain function. For $F_{\text {prob }}$, functions $f_{1}(x)=x, f_{2}(x)=1-$ $\frac{0.1}{x+1}$, and $f_{3}(x)=0.1 \cdot 10^{x}$ were tested. Usage of the last two functions leads to non-uniform splitting patterns. Usage of $f_{2}(x)$ leads to an earlier finish of the splitting process, though it requires setting a higher value of maximal tree depth. Conversely, $f_{3}(x)$ causes more sustainable splitting at the beginning than $f_{1}(x)$. It was experimentally shown that $f_{1}(x)$ and $f_{3}(x)$ are the best to satisfy the condition of normal distribution of tree widths.

We define an operation of computing cardinality for every node of the tree:

$$
M_{v}= \begin{cases}v_{\text {child }}[0]+v_{\text {child }}[1]+1 & \left|v_{\text {child }}\right|>0 \\ 1 & \left|v_{\text {child }}\right|=0\end{cases}
$$

As 1 denotes a computational node time unit, cardinality of a tree node characterizes its computational complexity.

For development, we used Python 3.7 programming language. From its point of view, a tree is a hierarchy of nested objects whose types are either 'list' or 'int'. If it is decided to stop splitting while creating a tree, the current vertex becomes a leaf, and in the Python structure it is represented as 1 . In the suggested simulator, 1 corresponds to an abstract unit of working time of a computational node.

It is worth noticing that criterion (1) does not guarantee that the maximum depth would be achieved in generation, which makes repetition of an experiment challenging.
Apart from that, the suggested Python implementation of a tree requires great amounts of memory and time expenses on generation if a processed tree has big depth. Building a tree during simulation by splitting a vertex when it is processed may be a possible solution for this problem, but it would decelerate the simulation dramatically. Apart from that, it would become difficult to evaluate computational complexities of branches.

Thus, it was proposed to omit the requirement of normal distribution of tree widths and generate child nodes according to the cardinality criterion. It is supposed that $K$ split iterations of a vertex with cardinality $M$ would result in $(K+1)$ child nodes with aggregated complexity $(M-K)$. The overall complexity is distributed among them randomly. Such a tree processing method has random nature as well, though virtual time averaging among multiple executions leads to the results that are close to the real systems. At the same time, real time expenses appear to be reasonably less than in the previous methods. Finally, memory expenses include only an integer value representing cardinality of the whole tree.

\section{Simulator Design}

The simulator workflow is based on algorithms suggested in $[12,13]$. The system consists of a manager process that creates tasks and aggregates computational results and $N$ computational nodes that perform computations simultaneously. A computational node and the manager node can exchange data through a communication channel. It should be highlighted that this use case is just a specific instance as many real systems may include several manager processes or have none of them at all $[14,15]$. The simulator can be modified to support different balancing algorithms. In particular, it would be more appropriate to use the same balancing function for all nodes.

States of both types of processes are described in [13]. In the initial step of the computational workflow, the man- 


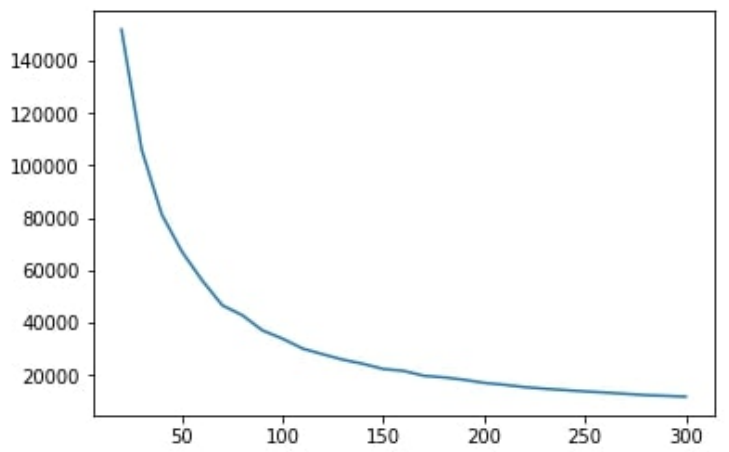

Figure 2: Virtual time of a subproblem tree processing for different numbers of computers, units

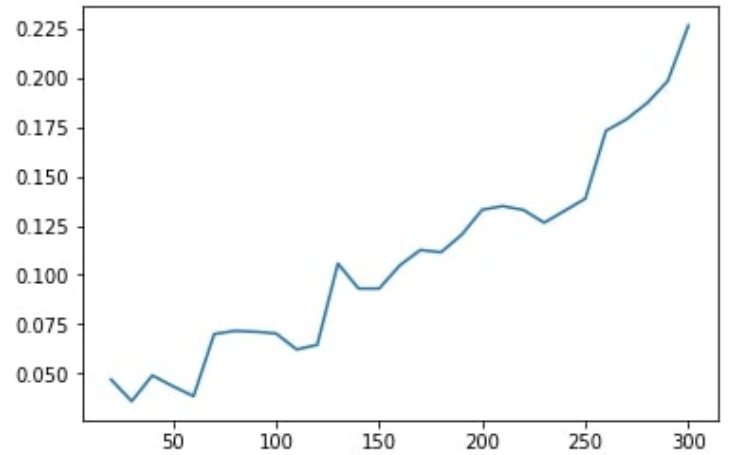

Figure 3: Real time spent for simulation with dynamic time steps, seconds

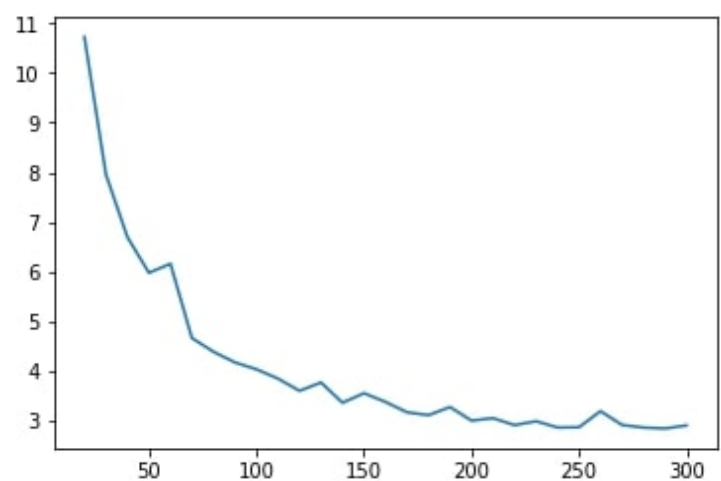

Figure 4: Real time spent for simulation with uniform time steps, seconds

ager process performs $K$ split iterations to create an initial set of tasks for computational nodes. Split is performed with breadth-first search (BFS) algorithm to maximize the number of created tasks.

Splitting with $B F S$ is modeled with tree cardinality evaluation. After $K$ iterations are performed, $(K+1)$ tasks with the cardinality sum equal to $\left(M_{\text {tree }}-K\right)$ are created.

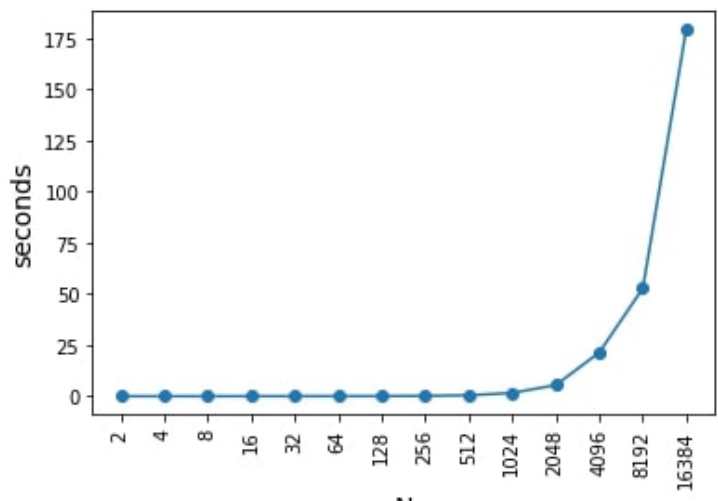

Figure 5: Real time scalability

The overall complexity is distributed among them randomly.

The created tasks are distributed uniformly between all computational processes. In order to avoid the situation when some nodes are idle, $K$ is set equal to the number of computational nodes in all experiments.

After the initial task set is created, they are sent to computational processes. For modeling this step, we set a coefficient $p=0.2$ that denotes a ratio of time for sending information about one task to a processor time unit. The manager process is able to communicate with one computational process only, so delays are simulated for other computational nodes. When a computer receives tasks, its computational load becomes equal to the sum of cardinalities of all tasks.

A simulation time step is set dynamically basing on remaining times for current states of all processes. The step is set equal to the minimal time value. This approach allows to avoid long simulation of task processing performed by computational nodes.

\section{Computational Experiments}

Simulator testing was performed with a machine with Intel Core i5 $1.8 \mathrm{GHz}$ processor and $8 \mathrm{~Gb}$ of RAM. The modeled systems contained the manager process and $N=\overline{5,19}$ computers. Computations were modeled for a tree with cardinality equal to 1700164 . Values of modeled time averaged over 100 runs are presented in Figure 2. It can be seen that the graph demonstrates a clear hyperbolic decrease as the number of computational units rises, which is close to real evaluations.

Due to the usage of a dynamic time step, it becomes possible to achieve nearly constant real simulation time 

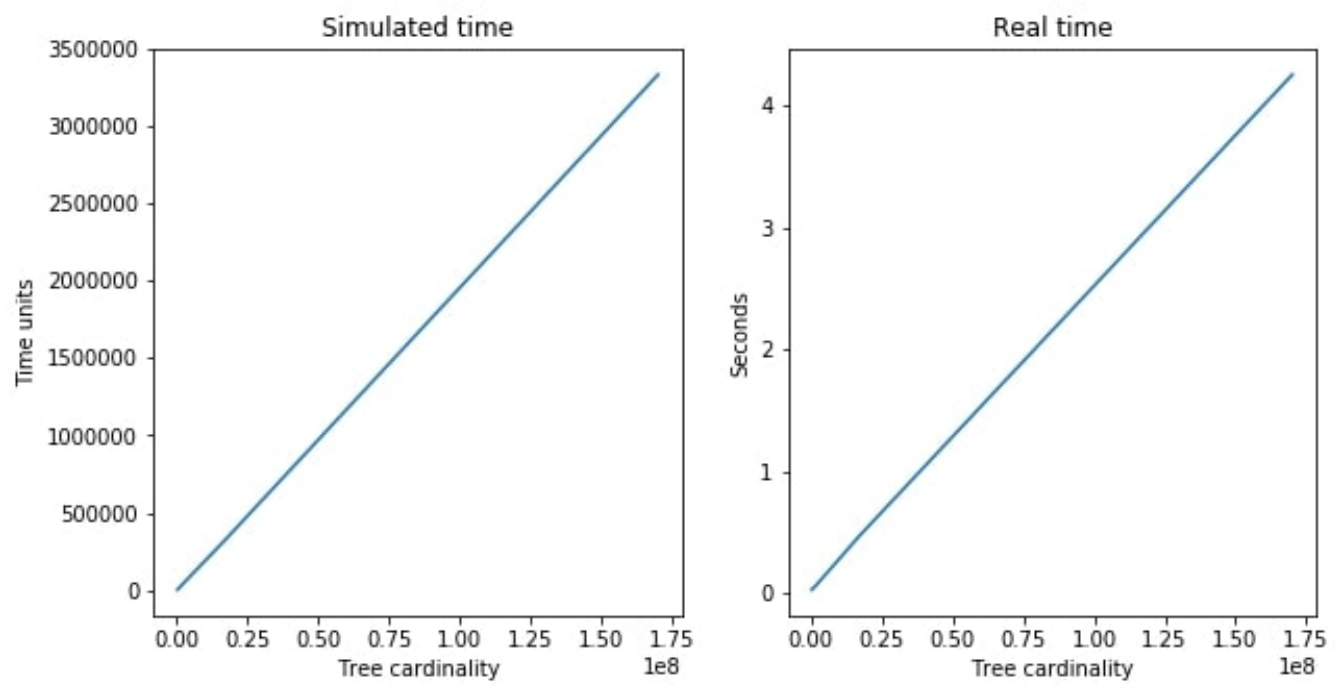

Figure 6: Simulated and real time values for different tree cardinalities with fixed $N=100$

independent of the number of computational nodes, as shown in Figure 3. It is worth noticing that the smallest time steps feature the period when tasks are sent to computational nodes. Time of this period depends on their number $N$ linearly, but for trees with high cardinality values this time is reasonably smaller than task processing period.

It can be seen from Figure 4 that in case of a uniform time step real time values reduce with the rise in the number of computational nodes. It can be explained by higher concurrency when the equal time step is substracted from a bigger number of time values. Conversely, real time measures for the dynamic step implementation increase, which is caused by the higher number of small time steps at the beginning of the simulation. These time steps represent the transition of created tasks to computational units. Figure 5 presents how real time spent on simulation depends on $N$. It can be seen that simulations with up to $2^{14}$ nodes can be processed with the current hardware in a reasonable time.

\section{Discussion}

Comparison of Figures 3 and 4 gives a clear idea about general superiority of the dynamic step approach over the the uniform one. However, modeling network performance decelerates the simulation reasonably at high values of $N$. Thus, the developed simulator requires a better modeling of network communications.

To prove the general consistency and robustness of the developed simulator, an experiment with scaling the tree cardinality was held. Figure 6 presents virtual and real time evaluations of that experiment. Tree cardinality was set as $M_{\text {tree }}=170016 \cdot 10^{i}, i=\overline{0,3}$, the number of computational units was set to $N=100$. Values in Figure 6 are averaged over 20 executions. It can be seen that the system demonstrates appropriate linear scalability both for the simulated and the real time.

\section{Conclusion}

The paper presents a parallel computations' simulator developed for problems solved by Branch and Bound method. Subdomain trees were analyzed, an algorithm of generating artificial trees of the same kind was suggested. Design of the simulator is described. The developed simulator uses dynamically set time steps, thanks to which it becomes possible to reduce the real time simulation evaluations reasonably. In the future, it can be modified for modeling advanced balancing techniques.

Acknowledgement: Partially supported by Program 2 of Presidium of RAS "Mechanisms for ensuring fault tolerance in modern high-performance and highly reliable computing”.

\section{References}

[1] Lawler E.L., Wood D.E., Branch-and-bound methods: A survey, Operations research, 14(4), 1966, 699-719 
[2] Evtushenko Y., Posypkin M., Sigal I., A framework for parallel large-scale global optimization, Computer Science-Research and Development, 23(3-4), 2009, 211-215

[3] Evtushenko Y., Posypkin M., Rybak L., Turkin A., Approximating a solution set of nonlinear inequalities, Journal of Global Optimization, 71(1), 2018, 129-145

[4] Kolpakov R.M., Posypkin M.A., Sigal I.K., On a lower bound on the computational complexity of a parallel implementation of the branch-and-bound method, Automation and Remote Control, 71(10), 2010, 2152-2161

[5] Kolpakov R., Posypkin M., The Scalability Analysis of a Parallel Tree Search Algorithm, in International Conference on Optimization and Applications, Springer, 2018, 186-201

[6] Bychkov I., Manzyuk M., Semenov A., Zaikin O., Afanasiev A., Posypkin M., Technology for integrating idle computing cluster resources into volunteer computing projects, in 2015 th International Workshop on Computer Science and Engineering: Information Processing and Control Engineering, WCSE 2015-IPCE, 2015

[7] Ignatov A., Posypkin M., BOINC-based Branch-and-Bound, in Russian Supercomputing Days, Springer, 2018, 511-522

[8] Carothers C.D., Bauer D., Pearce S., ROSS: A high-performance, low-memory, modular Time Warp system, Journal of Parallel and Distributed Computing, 62(11), 2002, 1648-1669
[9] Casanova H., Giersch A., Legrand A., Quinson M., Suter F., Versatile, Scalable, and Accurate Simulation of Distributed Applications and Platforms, Journal of Parallel and Distributed Computing, 74(10), 2014, 2899-2917

[10] Legrand A., Marchal L., Casanova H., Scheduling distributed applications: the simgrid simulation framework, in CCGrid 2003. 3rd IEEE/ACM International Symposium on Cluster Computing and the Grid, 2003. Proceedings., IEEE, 2003, 138-145

[11] Posypkin M., Usov A., Implementation and verification of global optimization benchmark problems, Open Engineering, 7(1), 2017, 470-478

[12] Golubeva Y., Orlov Y., Posypkin M., A tool for simulating parallel branch-and-bound methods, Open Engineering, 6(1), 2016

[13] Evtushenko Y., Golubeva Y., Orlov Y., Posypkin M., Using simulation for performance analysis and visualization of parallel Branch-and-Bound methods, in Russian Supercomputing Days, Springer, 2016, 356-368

[14] Gorchakov A., K-frontal method of nonuniform coverings, International Journal of Open Information Technologies, 7(8), 2019, 65-69

[15] Gorchakov A., Ignatov A., Malyshev D., Posypkin M., Parallel algorithm for approximating the work space of a robot, International Journal of Open Information Technologies, 7(1), 2019, 1-7 